\title{
Acute Renal Replacement Therapy in Pediatrics
}

\author{
Rajit K. Basu, ${ }^{1,2}$ Derek S. Wheeler, ${ }^{1,2}$ Stuart Goldstein,, ${ }^{2,3}$ and Lesley Doughty ${ }^{1,2}$ \\ ${ }^{1}$ Division of Critical Care and Center for Acute Care Nephrology, Cincinnati Children's Hospital Medical Center, Cincinnati, \\ OH 45229, USA \\ ${ }^{2}$ Department of Pediatrics, College of Medicine, University of Cincinnati, Cincinnati, OH 45229, USA \\ ${ }^{3}$ Division of Nephrology and Center for Acute Care Nephrology, Cincinnati Children's Hospital Medical Center, Cincinnati, \\ OH 45229, USA
}

Correspondence should be addressed to Rajit K. Basu, rajit.basu@cchmc.org

Received 14 February 2011; Accepted 4 April 2011

Academic Editor: Michel Fischbach

Copyright ( 2011 Rajit K. Basu et al. This is an open access article distributed under the Creative Commons Attribution License, which permits unrestricted use, distribution, and reproduction in any medium, provided the original work is properly cited.

\begin{abstract}
Acute kidney injury (AKI) independently increases morbidity and mortality in children admitted to the hospital. Renal replacement therapy (RRT) is an essential therapy in the setting of AKI and fluid overload. The decision to initiate RRT is complex and often complicated by concerns related to patient hemodynamic and thermodynamic instability. The choice of which RRT modality to use depends on numerous criteria that are both patient and treatment center specific. Surprisingly, despite decades of use, no randomized, controlled trial study involving RRT in pediatrics has been performed. Because of these factors, clear-cut consensus is lacking regarding key questions surrounding RRT delivery. In this paper, we will summarize existing data concerning RRT use in children. We discuss the major modalities and the data-driven specifics of each, followed by controversies in RRT. As no standard of care is in widespread use for RRT in AKI or in multiorgan disease, we conclude in this paper that prospective studies of RRT are needed to identify best practice guidelines.
\end{abstract}

\section{Introduction}

Acute kidney injury (AKI) affects a significant proportion of critically ill children. Using a revised AKI definition, a recent study indicated that up to $10 \%$ of all children admitted to the pediatric intensive care unit (PICU) suffer some degree of kidney injury [1]. The kidney is central to numerous homeostatic mechanisms in the body including: (1) fluid balance, (2) acid-base balance, (3) erythropoiesis, and (4) vascular tone regulation. Aberrancies in kidney function, therefore, can negatively affect host survival. AKI is often recognized as a complication of multi-organ disease and independently increases the risk of mortality [2,3]. Although formal and definite indications for use are lacking, RRT is employed for AKI, fluid overload, and sepsis. While revised definitions of AKI have aided in stratification of injury severity [4-6], controversy and uncertainty surrounds the use of RRT. It is unclear which patients are appropriate for therapy, which modalities should be used, what the triggers are for initiation, what "dose" should be prescribed, and how long treatment should continue.
The Prospective Pediatric Continuous Renal Replacement Therapy (ppCRRT) registry, a comprehensive and collaborative registry composed of thirteen select pediatric referral centers, was established in 2000 to prospectively evaluate clinical aspects of CRRT and recently reported an overall mortality of $43.1 \%$ for critically ill children placed on RRT [7]. This statistic underscores the need for urgent and broad-based prospective study of children placed on RRT. In this paper, we will lay a framework for understanding the rationale for RRT initiation, investigate the evidence for use of specific RRT modalities (Table 1), outcomes using these modalities, and also establish a purpose statement for the future of RRT study.

\section{The Different Modalities of Renal Replacement Therapy}

2.1. Peritoneal Dialysis. Peritoneal dialysis (PD) is the most widely available form of renal replacement therapy used in children. Almost all centers that care for acutely ill children 
TABLE 1: Comparison of peritoneal dialysis (PD), intermittent hemodialysis (IHD), and continuous renal replacement therapies (CRRT).

\begin{tabular}{lccc}
\hline Variable & PD & IHD & CRRT \\
\hline Continuous therapy & Yes & No & Yes \\
Hemodynamic stability & Yes & No & Yes \\
Fluid balance achieved & Yes/No, Cycle dependent & Yes/No, Intermittent & Yes, pump controlled \\
Ease of use & Yes & No & No \\
Adequate nutrition delivery & variable & variable & Yes \\
Solute control & Yes & Yes & Yes \\
Ultrafiltration control & Variable & Yes & Yes \\
Anticoagulation & No & Yes & Yes \\
Acute ingestion removal & No & Yes & Variable \\
Continuous toxin removal & Variable & Yes \\
ICU nursing needs & Low & High & High \\
Patient mobility & No & Yes & Ho \\
Cost & Low & High & Yes \\
Vascular access need & No & Yes & Yes \\
Infection potential & Yes & Yes & Yes \\
Use in inborn-errors of metabolism & No & \\
\hline
\end{tabular}

Adapted with permission from Walters et al. [8].

are capable of incorporating this technique into practice. PD is especially important for developing countries with limited resources.

Pro. The benefits of peritoneal dialysis include ease and quickness of catheter insertion, inexpensive cost compared to other modalities, and general tolerance with regards to hemodynamic stability. Percutaneously placed intraperitoneal catheters or permanent Tenckhoff catheters are used even in neonates, and catheter placement is relatively simple compared to the vascular access required for intermittent hemodialysis (IHD) or continuous renal replacement therapy (CRRT). Due to ease of tolerance, PD catheters have been prophylactically placed in neonates after cardiopulmonary bypass surgery felt to be at high risk for ischemic kidney injury [9]. Of global importance, particularly for areas with little technologic ability, PD can be performed manually and does not require a cycling device. Peritoneal dialysis fluid, either lactate based (USA) or bicarbonate based (outside USA), is nearly universally available. A major advantage of PD is absence of a need for anticoagulation. Functionally, PD is ideally suited for patients that have moderate illness and are poor candidates for modalities which require anticoagulation or large vascular access.

Con. Due to its relatively low clearance compared to IHD and CRRT, PD is ill suited for situations such as acute toxin ingestions, severe metabolic disturbances [10]. In cases of tremendous fluid overload, the rate of fluid removal of PD may not be rapid enough to prevent subsequent injury and morbidity. Manual PD can be labor intensive for the bedside practitioner, especially if the cycle frequency is high. Also, PD cannot be used in children with congenital abdominal malformations such as omphalocele, gastroschisis, and bladder exstrophy, or in patients with significant abdominal adhesions.

Evidence. Though rates of PD use have decreased in the PICU, it is still the most commonly used method of RRT in children in the world [11]. The use of PD is favored in the neonates who often have difficult vascular access, low tolerance to volume shifts, and thermodynamic instability [12]. In neonates with heart failure, PD provides a safe and adequate strategy for children and allows reasonable creatinine clearance rates [13]. The use of peritoneal dialysis is documented in children following cardiopulmonary bypass; Sorof et al. report no deaths or adverse hemodynamic effects during PD in 20 consecutive children immediately after cardiopulmonary bypass (CPB) [9, 13-17]. In a single center study, survivors after $\mathrm{CPB}$ had a shorter interval between the diagnosis of AKI and PD initiation than nonsurvivors $(1.2+0.4$ days versus $4.3+1.2$ days $)$ [17]. Numerous other reports demonstrate the safety and efficacy of PD after cardiopulmonary bypass including providing effective dialytic efficiency measurements $(K t / V>2.1$, where $K$ is the clearance of urea, $t$ is the time of dialysis, and $V$ is a patient's total body water; goal for PD is $>2.0 /$ week) [15-19].

Opinion. PD is the most often used form of RRT in adults and children worldwide, owing to cost and ease of use [20]. In skilled hands, PD is highly effective at removing solute and fluid and can be tailored to patient needs. Practitioners should be mindful of the potential for PD if vascular catheter placement is problematic. This modality does not require anticoagulation and is ideal for neonates, children with inadequate vascular access, and children who can tolerate slower fluid removal and electrolyte correction. 
2.2. Intermittent Hemodialysis. Intermittent hemodialysis (IHD) use requires center selective criteria of technical expertise and trained personnel and patient selective criteria of hemodynamic stability and vascular access.

Pro. The use of IHD is ideal for acute presentations of renal dysfunction or electrolyte imbalance. Because it offers the highest dose of dialysis in the shortest time frame, IHD is perfectly suited for disease processes which cause acute disruptions in homeostasis such as drug ingestions, tumor lysis syndrome, and hyperammonemia. Additionally, it allows for isolated ultrafiltration and titration of dialysis fluid solute concentration to correct metabolic disturbances such as hypernatremia. IHD is time limited, allowing for patient mobility, for example, to travel if needed for other diagnostic tests or procedures. In centers where continuous RRT is not available, some IHD machines permit slower and extended dialysis (SLED) which allows for gradual removal of solute and/or fluid [21].

Con. The technical requirements of IHD represent a major challenge to its use. IHD use is limited by the trained personnel available at a given medical care center and by the technical support available if trouble arises. Secondly, IHD requires vascular access in a large vessel. Though performed, this is especially problematic in children for whom obtaining access is a chronic problem [22]. Neonates may be unable to tolerate IHD simply because of inability to offer an appropriate cannula size to maintain adequate flow rates. Additionally, large IHD catheter placement can lead to vascular damage such as stenosis and thrombosis. Many nephrologists feel that for this reason, the subclavian veins should be avoided altogether [8]. Future chronic dialysis access needs may be greatly impeded by placement of temporary IHD catheters [23]. IHD use causes large volume shifts and may be unfeasible in patients that are hemodynamically unstable and those, such as small infants, who do not tolerate the volume shifts that occur during dialysis. Disequilibrium syndrome, secondary to rapid osmolar shifts, may complicate hemodialysis leading to seizures and cerebral edema, but it can be offset with short dialytic runs or with small doses of mannitol [24]. IHD requires anticoagulation, most commonly with heparin (although low-molecular-weight heparins and the alternative anticoagulants danaparoid, lepirudin, and citrate can be used in patients with heparin-induced thrombocytopenia). Finally, because it is time limited, IHD use does not lessen the fluid restrictions that are often placed on critically ill patients. Thus, using IHD requires diligent daily planning to balance UF with goal fluids, including boluses and blood products, and nutrition.

Evidence. Prior to the rise in popularity of continuous renal replacement therapy, intermittent hemodialysis was the modality of choice in patients requiring aggressive support for acute kidney injury. Other than for acute ingestions and electrolyte disturbances, the use of IHD has taken a backseat to CRRT in the intensive care setting requiring solute and fluid control. The adult SHARF 4 trials (Stuivenberg
Hospital Acute Renal Failure), however, found no differences in mortality or renal recovery in 316 adults with AKI between IHD and CRRT [25]. Similarly, a meta-analysis of 43 studies comparing IHD to CRRT in adults reports that CRRT does not offer an advantage with regard to survival or long term dialysis dependence [26]. Pediatric data comparing IHD to other modalities in AKI is limited. In 2001, Bunchman et al. published data on 226 children receiving RRT. In this study, $81 \%$ of the 61 children receiving IHD survived compared to only $40 \%$ for hemofiltration; however, the former group received significantly less vasopressor support than the latter [27]. Retrospectively, the survival of pediatric AKI for IHD (73-89\%) has been higher than those for CRRT (34-58\%) [27].

Opinion. Intermittent hemodialysis is effective therapy for acute ingestions, toxins, and fluid overload. IHD carries risks for line placement, technical difficulty in small patients and neonates, and complications with rapid shifts in fluids and electrolytes. IHD is ideal for patients with acute electrolyte abnormalities and those that would benefit from and can tolerate high doses of dialysis in a short time frame [28].

2.3. Continuous Renal Replacement Therapy. The use of continuous dialysis for AKI is widespread in PICUs. Arteriovenous hemofiltration was first described in the late 1970s and the 1980s, including in infants [29]. CRRT became popular in pediatric AKI in part because venovenous filtration machines, which offset the need for patient perfusionpressure-driven ultrafiltrate, eased the problem of low driving pressures in pediatric patients. Additionally, advances in CRRT technology allowed gravimetric and volumetric control of hemofiltration to allow for accurate ultrafiltration flows [30]. Still, while CRRT use expands, in both adults and pediatrics the risk-benefit ratio pits known risks to theoretical benefits. A major limitation to the standardization of CRRT use is the lack of prospective randomized data from controlled studies, evaluating the time of initiation, route of delivery, and efficacy. Retrospective data attempts to answer some of these questions.

Pro. The major advantage of CRRT is that it affords nearly continuous adjustment of ultrafiltration. This feature makes it well suited for patients with hemodynamic instability $[31,32]$. CRRT (and IHD) allows for ultrafiltration to be independently regulated from solute removal. This confers great benefit for patients with sensitive electrolyte needs and fluid states. Finally, fluid delivery does not require restriction while CRRT is in use, affording increased freedom for nutrition. The preferred modalities of CRRT in children are likely affected by site-specific familiarity and local preference, and data are retrospective $[8,22,31,33]$.

Con. The primary drawback to CRRT use is its complexity. The level of technical expertise required is high and, as such, is not available at many centers worldwide. The need for vascular access can often complicate its use. Attaining adequate flow rates in neonates and small children can be 
TABLE 2: Ideal Catheter size and patient size for CRRT.

\begin{tabular}{lcc}
\hline Patient size & Catheter size & Site of insertion \\
\hline Neonate & $7 \mathrm{Fr}$ & IJ/EJ, femoral \\
3-6 kilogram & $7 \mathrm{Fr}$ & IJ/EJ, femoral \\
6-10 kilogram & $8 \mathrm{Fr}$ & IJ/EJ, subclav, femoral \\
$>10-20$ kilogram & $9 \mathrm{Fr}$ & IJ/EJ, subclav, femoral \\
$>20-30$ kilogram & $10 \mathrm{Fr}$ & IJ/EJ, subclav, femoral \\
$>30$ kilogram & $12 \mathrm{Fr}$ & IJ/EJ, subclav, femoral \\
\hline
\end{tabular}

Fr: French; IJ: internal jugular vein; EJ: external jugular vein; subclav: subclavian vein. Adapted with permission from Goldstein [34].

difficult even despite recommended catheter size placement (Table 2). As in IHD, CRRT in small patients is complicated by the fact that a large percentage (10-15\%) of blood volume may be extracorporeal at any given time. This amount of volume shift can lead to a high amount of thermodynamic instability. Bradykinin release syndrome (hypotension, mucosal congestion, and bronchospasm upon contact of patient blood with a hemofilter) is a problem with CRRT in infants using an AN-69 membrane circuit. Finally, anticoagulation may pose added complications to patients in septic shock and disseminated intravascular coagulation (DIC).

Evidence. CRRT offers minute to minute control of ultrafiltrate and affords practitioners the flexibility to use volume intense medications, blood products, and nutrition. CRRT offers better control of uremia compared to PD or IHD [35] and allows practitioners to control solute levels to targets, which is critical in cases such as children with increased intracranial pressure who require tight control of serum osmolarity [36]. However, no prospective study of CRRT exists in either the adult or the pediatric literature. Also, no controlled trial comparing the efficacy of PD to IHD to CRRT in pediatrics exists. A study comparing PD to CRRT demonstrated a significant survival difference with CRRT $(85 \%)$ versus $\mathrm{PD}(43 \%)$, but critics have indicated that rigid tubing, manually exchanged fluid, and hyperglycemia in the PD group may have skewed the results $[37,38]$. A recent multicenter adult study in Belgium indicated that, in 1303 patients, mortality was higher (58\% versus $43 \%$ ) for AKI patients in the RRT group than in conservative management group despite correction for severity of illness [39]. Unfortunately, clear evidence supporting or contraindicating the use of CRRT in pediatrics is lacking. Fortunately, through the ppCRRT, the study of pediatric RRT has progressed and more data emerges regarding the confounding variables associated with the risks and benefits of RRT. Some of these variables are discussed below.

Opinion. As center-specific technical expertise expands, the use of CRRT continues to also expand. Despite limited data indicating its superiority over PD or IHD, the ability to finely control fluid balance and electrolyte derangements makes CRRT an ideal option in the hemodynamically unstable patient. Additionally, the ability to provide optimal nutrition and deliver blood products and medicine while on CRRT (without worrying excessively about fluid shifts) is highly desirable. The tight control of such deliverables is paramount in the care of the critically ill pediatric patient and the primary reason why CRRT use expands. Institutional capability, technical familiarity, and ability to obtain adequate vascular access are barriers to its use, but efficacy in unstable patients is an attractive quality in this modality.

\subsection{Controversies in Renal Replacement Therapy}

Initiation of RRT. In the only randomized trial of early versus late initiation of RRT in critically ill patients [40], 106 ventilated, oliguric adults were randomized to either "high volume-early" (initiation 8 hours after stratification, 72-96 L hemofiltration in 24 hours), "low volume-early" (initiation 8 hours after stratification, 24-36 L hemofiltration in 24 hours), and "low volume-late" (initiation 36 hours after stratification, 24-36 L hemofiltration in 24 hours) of CRRT. Median duration of renal failure and 28-day survival were not significantly affected by timing of initiation. However, other data suggesting that early treatment is superior to late treatment is encouraging in patients who developed AKI following CPB [41-44]. There is also evidence suggesting that early RRT affords critically ill patients with a therapeutic strategy that goes beyond organ support. In other words, RRT may a serve a more direct, therapeutic role in ameliorating renal injury and improving the chances of "renal recovery." Renal recovery is variably defined in the literature, though most definitions used include the criterion of freedom from chronic RRT $[45,46]$. The pediatric data regarding timing of initiation is largely retrospective and put into the context of triggers such as fluid overload and presence of sepsis.

Fluid Overload and CRRT. A retrospective study of 21 children receiving RRT for AKI suggested that the degree of fluid overload (FO) at time of RRT initiation was significantly lower in survivors than in nonsurvivors ( $16.4 \%$ versus $34 \%)$ [31]. In a larger study of 113 children with multiple-organ dysfunction syndrome (MODS) started on CRRT, median $\%$ FO was significantly lower in survivors compared to nonsurvivors $(7.8 \%$ versus $15.1 \%)$, and mortality related to FO was independent of severity of illness [47]. Another study showed that, in 297 patients, \% FO was again significantly lower in survivors versus nonsurvivors (12.5\% versus $23.0 \%)$ [48]. In a prospective, uncontrolled, observational study DiCarlo initiated CRRT for ten children with ARDS after BMT regardless of presence of AKI and demonstrated an $80 \%$ survival rate [49]. Of the available retrospective evidence, the mortality for children started on CRRT is 10$57.1 \%$. In select centers, the use of PD after cardiopulmonary bypass during the immediate postoperative period is routine [9]. The ppCRRT data suggests that a threshold may exist for initiation of CRRT based on FO to improve mortality, a threshold that appears to be independent of severity of illness [7]. The threshold, 10-15\% FO, however, has yet to be prospectively tested. Further, it has yet to be demonstrated 
that children placed on CRRT for AKI have better outcomes than those without such therapy.

Sepsis and CRRT. RRT for sepsis without AKI has been attempted in adults, with the thought that RRT could remove harmful circulating cytokines and inflammatory mediators, but trials have been underpowered and results regarding efficacy have been varied. While many inflammatory mediators can be removed using RRT, specifically CRRT, it is unknown how the amount removed compares to the serum concentration of such mediators and the significance of certain levels. No studies of RRT designed specifically to analyze its efficacy in pediatric sepsis, absent AKI, have been performed. A recent metaanalysis of the major adult CRRT studies, comparing the efficacy of "intensive-dose" CRRT ( $>40 \mathrm{~mL} / \mathrm{kg} / \mathrm{hr})$ to "less-intensive-dose" CRRT ( $<25 \mathrm{~mL} / \mathrm{kg} / \mathrm{hr})$, found no beneficial effect of intensive dose on mortality in sepsis and AKI [50]. While in vitro data continues to suggest that CRRT may offer benefit for immunomodulation in sepsis, clear clinical data are lacking. Accordingly, in adults and in children, the use of CRRT for immunomodulation is not currently recommended [51].

Access. While adequate venous access is paramount to effective IHD or CRRT, PD confers the advantage of not requiring large vessel access. Catheter placement in children increases risk for thrombosis and sclerosis, especially in the subclavian veins, and may be detrimental to obtaining future access. Data from the ppCRRT indicates that smaller catheters (5 and 7 French) and those placed in the femoral veins were associated with shorter circuit lives than larger catheters and those placed in the internal jugular veins [52]. Appropriate catheter sizes and placement are mutually dependent and can vary, but a starting framework is shown in Table 2 [30].

Anticoagulation. The absence of a need for anticoagulation is a major advantage for PD use. Heparin and citrate are the major anticoagulants used in IHD and CRRT; registry data reports equal circuit viability with both substances [53]. While citrate allows the patient to be free of heparin-induced systemic anticoagulation, it may require several additional solutions to be run concurrently with the circuit: anticoagulant dextrose solution A (ACD-A) [30] and a continuous calcium infusion (infused through separate central access) to prevent hypocalcemia which occurs when citrate binds ionized calcium. Higher citrate clearances or lower citrate delivery methods must be used to prevent citrate lock, defined as elevated serum total calcium but low serum ionized calcium, which results from increased citrate buffering of free calcium from decreased citrate metabolism.

Dose. The high dose of dialysis delivered in relation to time is an advantage of IHD over PD and CRRT. Much of the data regarding dose of RRT is retrospective and focuses on CRRT. An initial study reported mortality lessened with larger doses ( $\geq 35 \mathrm{~mL} / \mathrm{kg} / \mathrm{hr}$ ) than with smaller doses $(20 \mathrm{~mL} / \mathrm{kg} / \mathrm{hr}$ ) [54].
However, a large recent study with meticulous documentation of actual doses received demonstrated no improvement in kidney function or mortality outcome in adults receiving high-intensity CRRT $(35 \mathrm{~mL} / \mathrm{kg} / \mathrm{hr})$ versus low-intensity CRRT $(20 \mathrm{~mL} / \mathrm{kg} / \mathrm{hr})$ or intermittent hemodialysis [55]. The few outcomes studies performed in pediatrics investigating the effects of RRT dosage and modality are retrospective. The pPCRRT in 2007 demonstrated no difference in overall outcomes based on modality or dose of CRRT used [56].

Modality of CRRT. As mentioned before, CRRT is offered in multiple modalities differing based on primary principle of filtration used (convection or diffusion). While the ppCRRT reported that 344 children on CRRT in 13 Centers in the United States used CVVHD (venovenous hemodialysis) (48\%), CVVHDF (venovenous hemodiafiltration) (21\%), CVVHF (21\%) (venovenous hemofiltration), and SCUF (slow continuous ultrafiltration) (1\%), most centers only offer one modality [57]. As the names imply, the modalities differ based on the ability to incorporate one or both methods of filtration (convection $\rightarrow$ filtration based on hydrostatic pressure, diffusion $\rightarrow$ dialysis, particles move based on concentration gradient). Some practitioners feel that CVVHD is the optimal method by which to remove small molecules in children, but this is most likely influenced by site-specific preference (or availability) [58]. Though not yet rigorously proven, filter life may also be affected by modality. In isolated studies, dialytic modes of CRRT offer longer filter survival (12 hours-48 hours) than purely filtration modes [59]. In the most recent ppCRRT data, more survivors had convective RRT than nonsurvivors (61.0\% versus $43.0 \%$ ) [48]. The lack of formal trials comparing modality in pediatrics limits any formal recommendation on use in relation to patient outcomes.

Outcomes. Limited prospective data is available to compare morbidity and mortality between the three dialytic modes. In adults, a study performed in a developing country analyzed PD versus CRRT in infection-related AKI and found that CRRT was significantly superior to PD in all end-points tested (reduction of creatinine, resolution of acidosis) [37]. In children, the data is quite limited, retrospective, and often limited to specific disease processes, such as cardiopulmonary bypass [60]. Data from the ppCRRT indicates 58\% survival for all children placed on RRT, although high mortality rates (liver 69\%, pulmonary 55\%, and stem cell 55\%) seen in select transplant populations and children under 10 kilograms influence the data significantly.

\section{AKI, Renal Angina, and the Why}

The use of renal replacement therapy in pediatrics continues to blossom. It is uncertain whether the risks of dialysis, both mechanical and technical, outweigh the perceived benefits (improved renal recovery or reduced mortality). At present, the only proper way to answer the question would be to conduct a prospective, randomized trial comparing the different RRT modalities to no invasive therapy, which would 
be a trial loaded with ethical concerns. Further, if acute dialysis for AKI is actually beneficial, the critical point of intervention remains a mystery. Cardiac catheterization and coronary intervention therapy for myocardial infarction were optimized with the advent of troponins - tests made more reliable when run in patients with risk factors and signs of illness. Unfortunately, unlike chest pain, AKI does not hurt and the symptoms of AKI without fluid overload may be quite subtle. The phenotype of AKI in its earliest stage is unknown, which limits the applicability of biomarkers and detection strategies, and treatment efforts. Discovering the equivalent of angina for chest pain and myocardial infarction — renal angina-may strengthen biomarker utility, aid early AKI diagnosis, and possibly identify patients most likely to benefit from early renal replacement therapy [61]. Renal angina, defined as the composite of risk factors (i.e., high blood pressure, smoking for heart disease and shock, or sepsis for AKI) and subtle changes in renal function (small changes in creatinine clearance or fluid overload), may identify patients who are at the highest risk. Prospective trials comparing cohorts of patients and different modalities of RRT are needed to improve the delivery of care, and the ppCRRT registry aims to reach this goal.

\section{References}

[1] J. Schneider, R. Khemani, C. Grushkin, and R. Bart, "Serum creatinine as stratified in the RIFLE score for acute kidney injury is associated with mortality and length of stay for children in the pediatric intensive care unit," Critical Care Medicine, vol. 38, no. 3, pp. 933-939, 2010.

[2] S. Uchino, J. A. Kellum, R. Bellomo et al., "Acute renal failure in critically ill patients: a multinational, multicenter study," Journal of The American Medical Association, vol. 294, no. 7, pp. 813-818, 2005.

[3] S. Elapavaluru and J. A. Kellum, "Why do patients die of acute kidney injury?” Acta Clinica Belgica, no. 2, pp. 326-331, 2007.

[4] R. Bellomo, C. Ronco, J. A. Kellum, R. L. Mehta, and P. Palevsky, "Acute renal failure-definition, outcome measures, animal models, fluid therapy and information technology needs: the Second International Consensus Conference of the Acute Dialysis Quality Initiative (ADQI) Group," Critical Care, vol. 8, no. 4, pp. R204-R212, 2004.

[5] R. L. Mehta, J. A. Kellum, S. V. Shah et al., "Acute kidney injury network: report of an initiative to improve outcomes in acute kidney injury," Critical Care, vol. 11, no. 2, article R31, 2007.

[6] A. Akcan-Arikan, M. Zappitelli, L. L. Loftis, K. K. Washburn, L. S. Jefferson, and S. L. Goldstein, "Modified RIFLE criteria in critically ill children with acute kidney injury," Kidney International, vol. 71, no. 10, pp. 1028-1035, 2007.

[7] S. M. Sutherland, M. Zappitelli, S. R. Alexander et al., "Fluid overload and mortality in children receiving continuous renal replacement therapy: the prospective pediatric continuous renal replacement therapy registry," American Journal of Kidney Diseases, vol. 55, no. 2, pp. 316-325, 2010.

[8] S. Walters, C. Porter, and P. D. Brophy, "Dialysis and pediatric acute kidney injury: choice of renal support modality," Pediatric Nephrology, vol. 24, no. 1, pp. 37-48, 2009.

[9] J. M. Sorof, D. Stromberg, E. D. Brewer, T. F. Feltes, and C. D. Fraser, "Early initiation of peritoneal dialysis after surgical repair of congenital heart disease," Pediatric Nephrology, vol. 13, no. 8, pp. 641-645, 1999.

[10] F. Schaefer, E. Straube, J. Oh, O. Mehls, and E. Mayatepek, "Dialysis in neonates with inborn errors of metabolism," Nephrology Dialysis Transplantation, vol. 14, no. 4, pp. 910918, 1999.

[11] J. Cerdá, A. Bagga, V. Kher, and R. M. Chakravarthi, "The contrasting characteristics of acute kidney injury in developed and developing countries," Nature Clinical Practice Nephrology, vol. 4, no. 3, pp. 138-153, 2008.

[12] S. Subramanian, R. Agarwal, A. K. Deorari, V. K. Paul, and A. Bagga, "Acute renal failure in neonates," Indian Journal of Pediatrics, vol. 75, no. 4, pp. 385-391, 2008.

[13] S. Morelli, Z. Ricci, L. Di Chiara et al., "Renal replacement therapy in neonates with congenital heart disease," Contributions to Nephrology, vol. 156, pp. 428-433, 2007.

[14] Z. Ricci, S. Morelli, C. Ronco et al., "Inotropic support and peritoneal dialysis adequacy in neonates after cardiac surgery," Interactive Cardiovascular and Thoracic Surgery, vol. 7, no. 1, pp. 116-120, 2008.

[15] K. L. McNiece, E. E. Ellis, J. J. Drummond-Webb, E. E. Fontenot, C. M. O'Grady, and R. T. Blaszak, "Adequacy of peritoneal dialysis in children following cardiopulmonary bypass surgery," Pediatric Nephrology, vol. 20, no. 7, pp. 972976, 2005.

[16] M. C. Lin, Y. C. Fu, L. S. Fu, S. L. Jan, and C. S. Chi, "Peritoneal dialysis in children with acute renal failure after open heart surgery," Acta Paediatrica Taiwanica, vol. 44, no. 2, pp. 89-92, 2003.

[17] J. C. Chien, BE. T. Hwang, Z. C. Weng, L. C. C. Meng, and P. C. Lee, "Peritoneal dialysis in infants and children after open heart surgery," Pediatrics and Neonatology, vol. 50, no. 6, pp. 275-279, 2009.

[18] K. L. Chan, P. Ip, C. S. W. Chiu, and Y. F. Cheung, "Peritoneal dialysis after surgery for congenital heart disease in infants and young children," Annals of Thoracic Surgery, vol. 76, no. 5, pp. 1443-1449, 2003.

[19] J. E. Kist-van Holthe tot Echten, C. A. Goedvolk, M. B. M. E. Doornaar et al., "Acute renal insufficiency and renal replacement therapy after pediatric cardiopulmonary bypass surgery," Pediatric Cardiology, vol. 22, no. 4, pp. 321-326, 2001.

[20] J. Cerdá, A. Bagga, V. Kher, and R. M. Chakravarthi, "The contrasting characteristics of acute kidney injury in developed and developing countries," Nature Clinical Practice Nephrology, vol. 4, no. 3, pp. 138-153, 2008.

[21] A. N. Berbece and R. M. A. Richardson, "Sustained lowefficiency dialysis in the ICU: cost, anticoagulation, and solute removal," Kidney International, vol. 70, no. 5, pp. 963-968, 2006.

[22] J. M. Symons, P. D. Brophy, M. J. Gregory et al., "Continuous renal replacement therapy in children up to $10 \mathrm{~kg}$," American Journal of Kidney Diseases, vol. 41, no. 5, pp. 984-989, 2003.

[23] A. B. Lumsden, M. J. MacDonald, R. C. Allen, and T. F. Dodson, "Hemodialysis access in the pediatric patient population," American Journal of Surgery, vol. 168, no. 2, pp. 197-201, 1994.

[24] A. I. Arieff, "Dialysis disequilibrium syndrome: current concepts on pathogenesis and prevention," Kidney International, vol. 45, no. 3, pp. 629-635, 1994.

[25] R. L. Lins, M. M. Elseviers, P. Van der Niepen et al., "Intermittent versus continuous renal replacement therapy for 
acute kidney injury patients admitted to the intensive care unit: results of a randomized clinical trial," Nephrology Dialysis Transplantation, vol. 24, no. 2, pp. 512-518, 2009.

[26] N. Ghahramani, S. Shadrou, and C. Hollenbeak, "A systematic review of continuous renal replacement therapy and intermittent haemodialysis in management of patients with acute renal failure," Nephrology, vol. 13, no. 7, pp. 570-578, 2008.

[27] T. E. Bunchman, K. D. McBryde, T. E. Mottes, J. J. Gardner, N. J. Maxvold, and P. D. Brophy, "Pediatric acute renal failure: outcome by modality and disease," Pediatric Nephrology, vol. 16, no. 12, pp. 1067-1071, 2001.

[28] S. P. Andreoli, "Acute renal failure in the newborn," Seminars in Perinatology, vol. 28, no. 2, pp. 112-123, 2004.

[29] C. Ronco, A. Brendolan, and L. Bragantini, "Treatment of acute renal failure in newborns by continuous arterio-venous hemofiltration," Kidney International, vol. 29, no. 4, pp. 908915, 1986.

[30] S. L. Goldstein, "Overview of pediatric renal replacement therapy in acute kidney injury," Seminars in Dialysis, vol. 22, no. 2, pp. 180-184, 2009.

[31] S. L. Goldstein, H. Currier, J. M. Graf, C. C. Cosio, E. D. Brewer, and R. Sachdeva, "Outcome in children receiving continuous venovenous hemofiltration," Pediatrics, vol. 107, no. 6, pp. 1309-1312, 2001.

[32] S. L. Goldstein, M. J. G. Somers, P. D. Brophy et al., "The prospective pediatric continuous renal replacement therapy (ppCRRT) registry: design, development and data assessed," International Journal of Artificial Organs, vol. 27, no. 1, pp. 9 14, 2004.

[33] T. E. Bunchman, P. D. Brophy, and S. L. Goldstein, "Technical considerations for renal replacement therapy in children," Seminars in Nephrology, vol. 28, no. 5, pp. 488-492, 2008.

[34] S. L. Goldstein, "Overview of pediatric renal replacement therapy in acute kidney injury," Seminars in Dialysis Journal, vol. 22, no. 2, pp. 180-184, 2009.

[35] W. R. Clark, B. A. Mueller, K. J. Alaka, and W. L. Macias, "A comparison of metabolic control by continuous and intermittent therapies in acute renal failure," Journal of The American Society of Nephrology, vol. 4, no. 7, pp. 1413-1420, 1994.

[36] R. D. Swartz, R. T. Bustami, J. M. Daley, B. W. Gillespie, and F. K. Port, "Estimating the impact of renal replacement therapy choice on outcome in severe acute renal failure," Clinical Nephrology, vol. 63, no. 5, pp. 335-345, 2005.

[37] N. H. Phu, T. T. Hien, N. T. H. Mai et al., "Hemofiltration and peritoneal dialysis in infection-associated acute renal failure in vietnam," New England Journal of Medicine, vol. 347, no. 12, pp. 895-902, 2002.

[38] J. T. Daugirdas, "Peritoneal dialysis in acute renal failure-why the bad outcome?" New England Journal of Medicine, vol. 347, no. 12, pp. 933-935, 2002.

[39] M. M. Elseviers, R. L. Lins, P. Van der Niepen et al., "Renal replacement therapy is an independent risk factor for mortality in critically ill patients with acute kidney injury," Critical Care, vol. 14, no. 6, article R221, 2010.

[40] C. S. C. Bouman, H. M. Oudemans-van Straaten, J. G. P. Tijssen, D. F. Zandstra, and J. Kesecioglu, "Effects of early high-volume continuous venovenous hemofiltration on survival and recovery of renal function in intensive care patients with acute renal failure: a prospective, randomized trial," Critical Care Medicine, vol. 30, no. 10, pp. 2205-2211, 2002.
[41] U. Demirkiliç, E. Kuralay, M. Yenicesu et al., "Timing of replacement therapy for acute renal failure after cardiac surgery," Journal of Cardiac Surgery, vol. 19, no. 1, pp. 17-20, 2004.

[42] M. M. Elahi, M. Yann Lim, R. N. Joseph, R. R. V. Dhannapuneni, and T. J. Spyt, "Early hemofiltration improves survival in post-cardiotomy patients with acute renal failure," European Journal of Cardio-thoracic Surgery, vol. 26, no. 5, pp. 10271031, 2004.

[43] P. Bent, H. K. Tan, R. Bellomo et al., "Early and intensive continuous hemofiltration for severe renal failure after cardiac surgery," Annals of Thoracic Surgery, vol. 71, no. 3, pp. 832$837,2001$.

[44] S. V. Baudouin, J. Wiggins, B. F. Keogh, C. J. Morgan, and T. W. Evans, "Continuous veno-venous haemofiltration following cardio-pulmonary bypass. Indications and outcome in 35 patients," Intensive Care Medicine, vol. 19, no. 5, pp. 290-293, 1993.

[45] S. M. Bagshaw, "Epidemiology of renal recovery after acute renal failure," Current Opinion in Critical Care, vol. 12, no. 6, pp. 544-550, 2006.

[46] S. M. Bagshaw, "The long-term outcome after acute renal failure," Current Opinion in Critical Care, vol. 12, no. 6, pp. 561-566, 2006.

[47] J. A. Foland, J. D. Fortenberry, B. L. Warshaw et al., "Fluid overload before continuous hemofiltration and survival in critically ill children: a retrospective analysis," Critical Care Medicine, vol. 32, no. 8, pp. 1771-1776, 2004.

[48] S. M. Sutherland, M. Zappitelli, S. R. Alexander et al., "Fluid overload and mortality in children receiving continuous renal replacement therapy: the prospective pediatric continuous renal replacement therapy registry," American Journal of Kidney Diseases, vol. 55, no. 2, pp. 316-325, 2010.

[49] J. V. DiCarlo, S. R. Alexander, R. Agarwal, and J. D. Schiffman, "Continuous veno-venous hemofiltration may improve survival from acute respiratory distress syndrome after bone marrow transplantation or chemotherapy," Journal of Pediatric Hematology, vol. 25, no. 10, pp. 801-805, 2003.

[50] Z. Zhongheng, X. U. Xiao, and Z. Hongyang, "Intensive- vs less-intensive-dose continuous renal replacement therapy for the intensive care unit-related acute kidney injury: a metaanalysis and systematic review," Journal of Critical Care, vol. 25, no. 4, pp. 595-600, 2010.

[51] L. Brochard, F. Abroug, M. Brenner et al., "An official ATS/ERS/ESICM/SCCM/SRLF statement: prevention and management of acute renal failure in the ICU patient: an international consensus conference in intensive care medicine," American Journal of Respiratory and Critical Care Medicine, vol. 181, no. 10, pp. 1128-1155, 2010.

[52] R. Hackbarth, T. E. Bunchman, A. N. Chua et al., "The effect of vascular access location and size on circuit survival in pediatric continuous renal replacement therapy: a report from the PPCRRT registry," International Journal of Artificial Organs, vol. 30, no. 12, pp. 1116-1121, 2007.

[53] P. D. Brophy, M. J. G. Somers, M. A. Baum et al., "Multicentre evaluation of anticoagulation in patients receiving continuous renal replacement therapy," Nephrology Dialysis Transplantation, vol. 20, no. 7, pp. 1416-1421, 2005.

[54] C. Ronco, R. Bellomo, P. Homel et al., "Effects of different doses in continuous veno-venous haemofiltration on outcomes of acute renal failure: a prospective randomised trial," Lancet, vol. 356, no. 9223, pp. 26-30, 2000. 
[55] P. M. Palevsky, J. H. Zhang, T. Z. O'Connor et al., "Intensity of renal support in critically ill patients with acute kidney injury," New England Journal of Medicine, vol. 359, no. 1, pp. 7-20, 2008.

[56] J. Cerdá and C. Ronco, "Modalities of continuous renal replacement therapy: technical and clinical considerations," Seminars in Dialysis, vol. 22, no. 2, pp. 114-122, 2009.

[57] J. M. Symons, A. N. Chua, M. J. G. Somers et al., "Demographic characteristics of pediatric continuous renal replacement therapy: a report of the prospective pediatric continuous renal replacement therapy registry," Clinical Journal of The American Society of Nephrology, vol. 2, no. 4, pp. 732-738, 2007.

[58] D. Parakininkas and L. A. Greenbaum, "Comparison of solute clearance in three modes of continuous renal replacement therapy," Pediatric Critical Care Medicine, vol. 5, no. 3, pp. 269-274, 2004.

[59] J. Del Castillo, J. López-Herce, E. Cidoncha et al., "Circuit life span in critically ill children on continuous renal replacement treatment: a prospective observational evaluation study," Critical Care, vol. 12, article R93, 2008.

[60] F. Fleming, D. Bohn, H. Edwards et al., "Renal replacement therapy after repair of congenital heart disease in children: a comparison of hemofiltration and peritoneal dialysis," Journal of Thoracic and Cardiovascular Surgery, vol. 109, no. 2, pp. 322-331, 1995.

[61] S. L. Goldstein and L. S. Chawla, "Renal angina," Clinical Journal of The American Society of Nephrology, vol. 5, no. 5, pp. 943-949, 2010. 


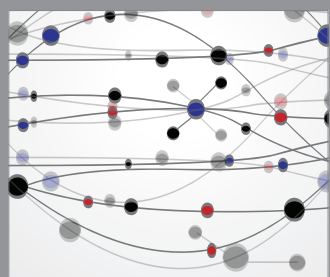

The Scientific World Journal
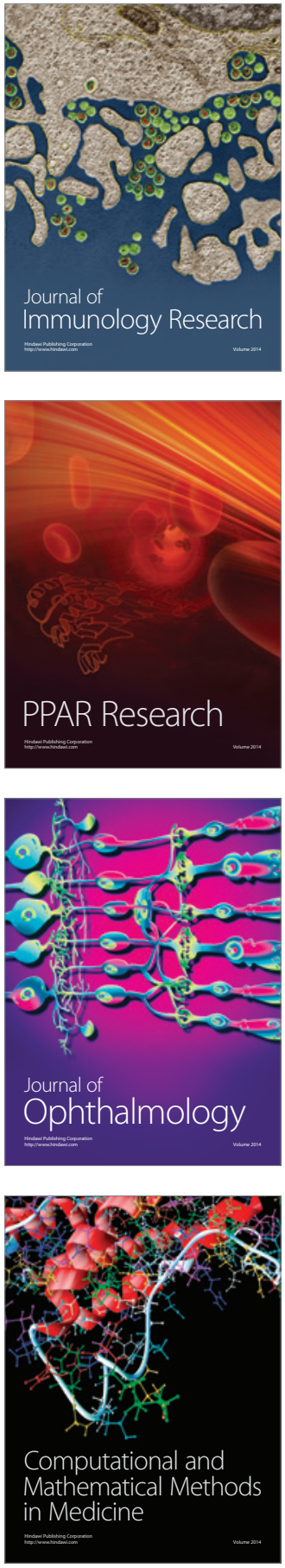

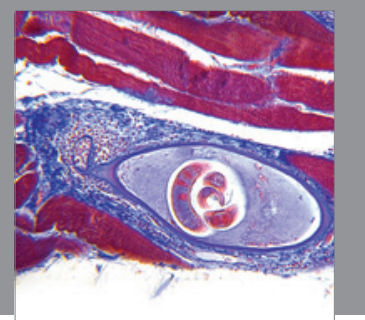

Gastroenterology

Research and Practice
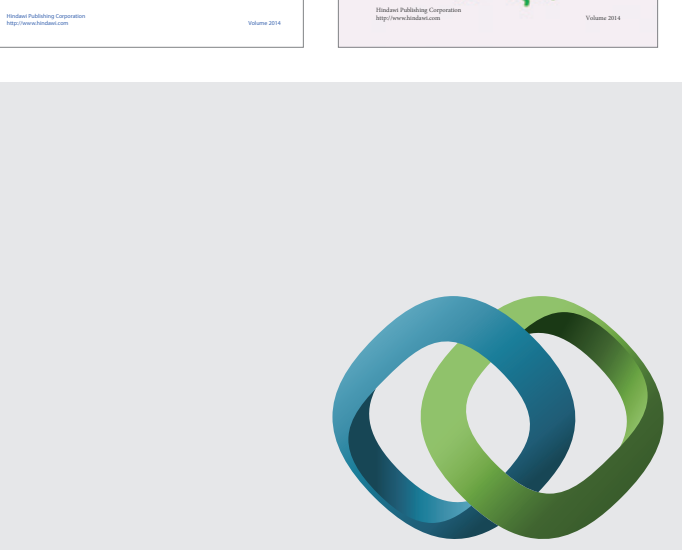

\section{Hindawi}

Submit your manuscripts at

http://www.hindawi.com
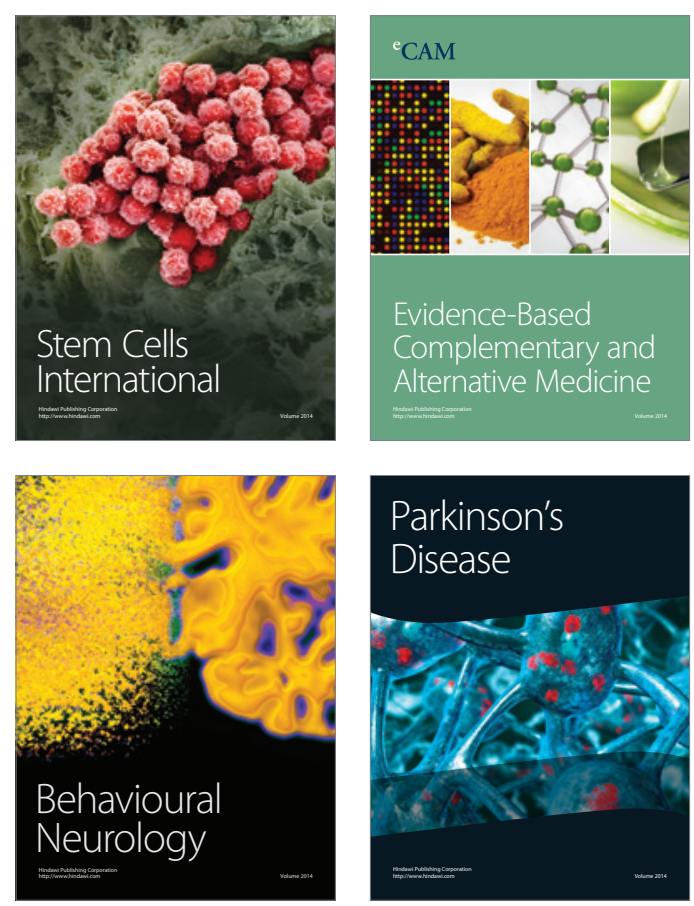

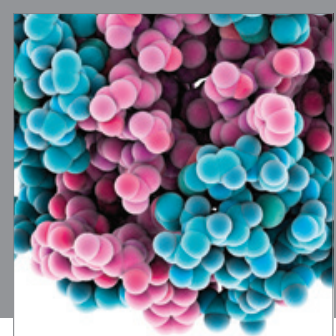

Journal of
Diabetes Research

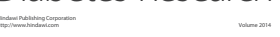

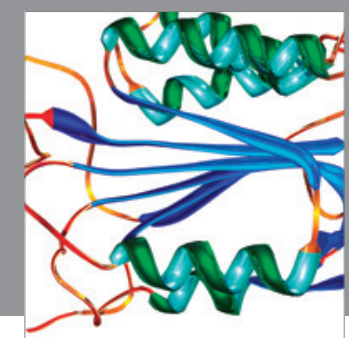

Disease Markers
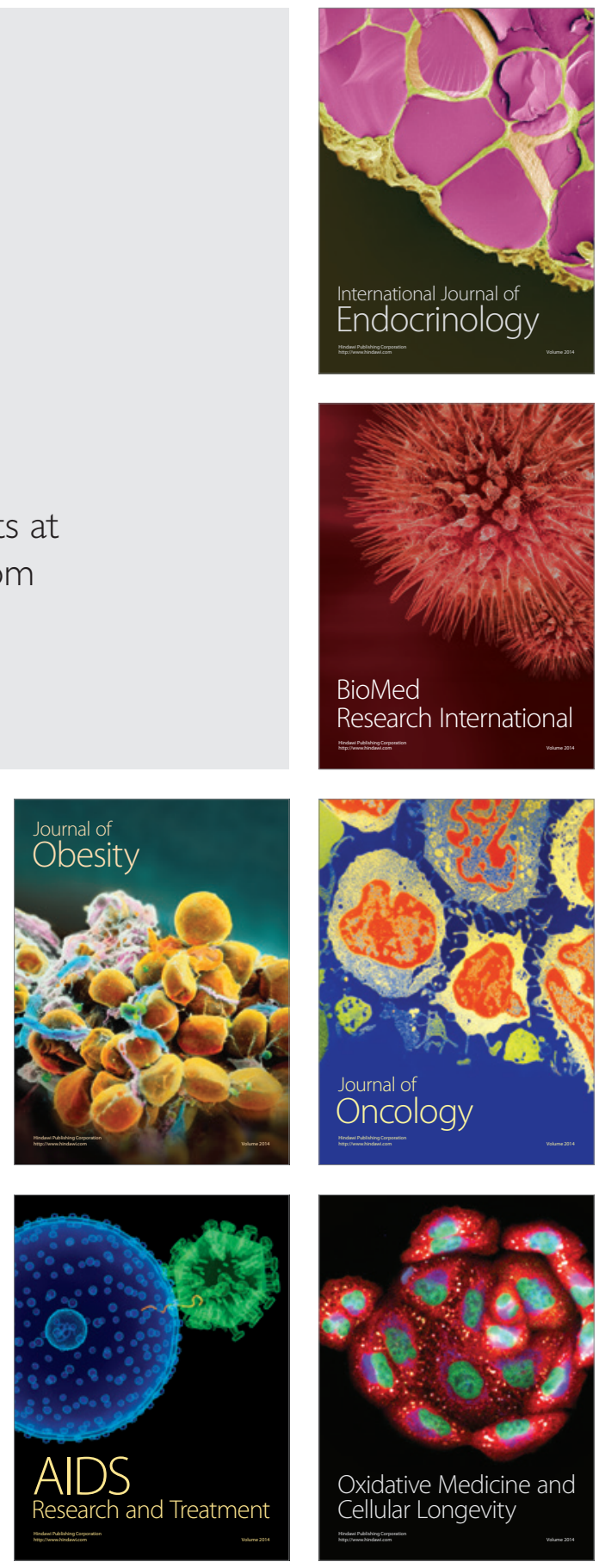